\title{
FUNDAMENTACIÓN EPISTEMOLÓGICA DE LOS DISEÑOS DE INVESTIGACIÓN NATURALISTA II. LOS ESTUDIOS DE CASOS DE ENFERMERÍA A PARTIR DEL ANÁLISIS DE TEXTOS DESDE LA TEORÍA DE LA ACCIÓN COMUNITARIA
}

ANA M. PIERA ORTS

Lic. CC. de la Educación.

Hospital General Universitario de Valencia

Correspondencia: Escalante, 18-3 $3^{\circ}-6^{a} .46011$ Valencia

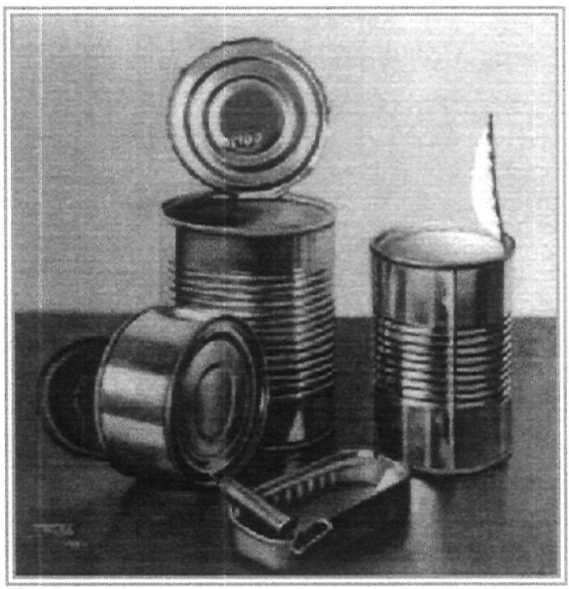

\section{RESUMEN}

Se presenta una fundamentación de los modelos de investigación cualitativa que se instalan bajo la modalidad de los estudios de casos, señalando las posibilidades que ofrece al investigador, al menos virtual, desde la óptica de la teoría de la acción comunicativa como propuesta para una indagación basada en observaciones a partir de datos dependientes del lenguaje .

\section{EPISTEMOLOGIC FOUNDAMENTATION OF THE DESING NATURALISTIC RESEARCH}

\section{THE ANALYSIS OF TEXT FROM THE THEORY COMMUNICATIVE ACTION IN NURSING CASES STUDIES}

SUMMARY

It in presented a explanation of qualitative research patterns with are installed under the variety of the cases studies marking the possibilities that it offers to the investigator, at least virtual, from the point of wiew of the communicative action theory as proposal for a search based on observations starting from facts dependens of the language. 


\section{INTRODUCCIÓN}

Dentro del ámbito de las investigaciones sociales, léase entornos sociológicos en los que se engloban instituciones de carácter político gubernamental, administrativo, educativo, sanitario, cultural, etc., se plantea la cuestión de la validez de sus conclusiones derivada de su universalidad. Sin embargo, tal validez vendría a justificarse a partir de la comprobabilidad del conocimiento científico una vez que hubiese sido comprobada la hipótesis, que tras la aplicación lógico deductiva, permitiese la derivación de enunciados singulares observables, cuya falsedad refutaría la hipótesis. Todo ello, atendiendo a que la investigación científica, sea de carácter social o no, tiene por objeto la búsqueda de leyes que puedan funcionar como premisas en explicaciones y predicciones deductivas. Si las leyes son conocidas y las condiciones materiales lo son igualmente, entonces un suceso puede predecirse, empleando la argumentación de tipo deductivo. Ahora bien, si nos planteamos la cuestión de "valor", a la hora de la recogida de los datos dentro en un entorno institucional, cuya aparente funcionalidad deja unos resquicios que confieren a la propia institución un carácter instituyente (ARDOINO, 1980), en el que los miembros pertenecientes a la misma, mantienen una comunicación que se inscribe dentro del "mundo de la vida", nos encontramos en las puertas de un debate controvertido que viene desarrollándose desde 1963 (HABERMAS, 1990a). En este debate se han discutido aspectos relativos a la naturaleza y comprensión interpretativa, tanto desde la perspectiva de la existencia de leyes generales, como de la naturaleza de la explicación, su relación con los valores o el acceso a los datos. Todo ello, apuntando siempre hacia la búsqueda de argumentos suficientes que permitiesen contar en la investigación social, con procedimientos capaces de captar el sentido de los fenómenos sociales, defendiendo así, el carácter distintivo de las ciencias sociales. Igualmente se han planteado cuestiones, partiendo de la defensa de la unidad metodológica de la ciencia, atendiendo a esquemas de comprobación de hipótesis como hemos señalado líneas arriba, tasando a la baja el papel del verstehen (comprensión simbólica y de sentido) en la lógica de las ciencias sociales, considerando este procedimiento como acientífico o precientífico; una forma de analizar este esquema como un modelo heurístico de búsqueda, que se ubica más bien en el contexto del descubrimiento que no en el de validación. Ante esta controversia se pueden adoptar una serie de posturas cuya fundamentación epistemológica, es sin duda una cuestión paradigmática, que, en definitiva, será a la que informe sobre una u otra metodología.

\section{ALGUNOS PLANTEAMIENTOS METODOLÓGICOS PLANTEADOS POR HABERMAS}

Habermas así como la mayoría de los integrantes de la Escuela de Francfort, mantiene posiciones contrarias al positivismo, considerando entre otras muchas cuestiones que "si de la virtud de su rigor positivista la ciencia hace la necesidad de relegar las cuestiones prácticas de la espontaneidad natural o a la arbitrariedad de juicios de valor incontrolados, entonces tampoco será posible esperar la ilustración de una opinión pública manifestada en forma políticamente madura" (HABERMAS, 1987, p. 348), y dice esto, refiriéndose a la formación universitaria cuando se plantea la transformación académica en su país, añadiendo que sólo a través de la autorreflexión de las ciencias, podremos constatar el contenido subyacente al interés por la libertad de la enseñanza e investigación "pues en esta dimensión es posible descubrir también las implicaciones sociales de un proceso de investigación inmanente en su apariencia" (HABERMAS, 1987a, pág. 357).

En este artículo nos detendremos en una fundamentación, que sin rehuir el conflicto de validez que ello podría suponer dentro del contexto científicoacadémico, parte de la relevancia valorativa que no solamente afecta al objeto de investigación, sino también al proceso de formación de conceptos. Un ejemplo de la relevancia de la valoración se puede hacer explícito, en la medida que la situación histórica del investigador, así como el punto de vista que guía su investigación no son fijos, en tanto que cuando trata de poner en orden sus ideas, la construcción de las categorías depende sustantivamente, de la forma en que articule el problema que se está estudiando. Tratando de ubicar la cuestión metodológica, habríamos de describir el modelo de análisis o paradigma particular en el que cabe ubicarse, para llevar a cabo el análisis o reflexión de las construcciones teóricas, que a partir del estudio de un 
hecho, o de un caso particular, podría ser asumido desde la perspectiva sociológica fenomenológica, desde la monológica de la fenomenología trascendental o desde la etnometodológica. Estas tres perspectivas, se corresponderían con una atención al mundo cotidiano de las experiencias, en el primer caso; con la situación biográfica de los sujetos, desde la segunda perspectiva; y por último, con las prácticas situadas de los miembros que generan para estos y para los observadores, el sentido que tienen las estructuras sociales objetivas, respectivamente.

En general la perspectiva fenomenológica, subordina el análisis de las estructuras sociales al análisis de las percepciones o interpretaciones de éstas (tales como roles, instituciones, normas, valores, sistemas y tradiciones culturales...). Cabe afirmar, que la comprensión de la motivación, puede quedar dentro del paradigma empírico lógico, sin trastornar su metodología y cuyas leyes de explicación, aparecen carentes de sentido a la hermenéutica. Pero de todos modos, en el avance de las ciencias sociales, vemos que aparecen tanto procedimientos de carácter empíricológicos como hermenéuticos, aunque se rechacen entre si como dos marcos intencionales cognoscentes $\mathrm{y}$, en ambos procedimientos, el sujeto cognoscente se halla vinculado intuitivamente al ámbito de los objetos investigados; pudiendo obtenerse de la investigación diferente tipo de datos, tanto pronósticos condicionados, como la clarificación de la autocomprensión de los sujetos cognoscentes o sus grupos sociales de referencia, a partir de cuyos resultados indistintamente, puede ejercerse el control de ámbitos sociales administrados.

En cualquier caso, la conexión entre el procedimiento metodológico de la investigación y la función del uso de los resultados de éste, se clarificará si se hacen explícitos los intereses que guían a los enfoques metodológicos; y en el caso de los analíticocausales e interpretativos, "el conocimiento termina en la elucidación de un sentido, de una significación práctica, es decir, en un tornar consciente. Con vistas a este fin, ahora es al procedimiento de la explicación y no al de la comprensión al que se atribuye un status metodológicamente subordinado (...) y sólo... podrá hallarse una respuesta precisa a la cuestión cuando las ciencias sociales en su es- tructura interna sirvan a la autocomprensión y a la ilustración" (HABERMAS, 1990a, p, 94).

En esta línea plantea que el científico social, en su calidad de observador, recoge datos dependientes del lenguaje por lo que ha de tener un status similar al del lego, al tiempo que sólo es factible que se abran al sujeto los significados si es participante, al menos virtual, en las comunicaciones de los miembros donde éstas se producen. Si ello no se produce de ese modo, no ha lugar al acceso posible a los nexos internos de sentido (HABERMAS, 1988), lo que viene a exigir que el investigador social sea miembro integrante del grupo donde se producen las acciones comunicativas.

Por ello, este autor señala que la problemática relación de explicación y comprensión en las ciencias sociales, no sólo está referida a los procedimientos y a los fines de las ciencias sociales, sino también a sus presupuestos epistemológicos y apunta que,

"La lógica de la ciencia, al reflexionar sobre las condiciones de las investigaciones posibles, cumple a propósito o inconscientemente la tarea de una interpretación previa a la realidad. En cambio, la expectativa de que la propia metodología hubiera de compartir la actitud de las ciencias y asumir la función de una ciencia auxiliar en punto a estrategias y tecnologías de investigación, refleja ya un prejuicio positivista. En la medida en que la metodología hace suyo este prejuicio reconoce el criterio que la crítica de Lazarsfeld aplica, y procede en términos instrumentalistas. Mi propósito, en cambio, es discutir las cuestiones metodológicas en actitud reflexiva" (HABERMAS, 1990a, p.126).

y recogiendo una afirmación de McCarthy, "como ha demostrado la historia de la ciencia, no existe una vía directa de las experiencias perceptivas a las construcciones teóricas que esté garantizada contra todo riesgo de extravío. Incluso los componentes más elementales de la "base evidencial" están interpretados categorialmente ("cargados de teoría") y por tanto también están sujetos a examen, revisión y rechazo" (McCARTHY, 1987, p.349).

Estas afirmaciones habermasianas, nos llevan aparentemente a un callejón sin salida, en el que no parece vayamos a poder encontrar una metodología que permita vislumbrar las condiciones bajo las que 
los enunciados son verdaderos, llevándonos hacia un modelo de análisis que se sitúa en el terreno de lo utópico, a partir de una situación ideal del habla.

Pero su análisis nos propone un tipo de investigación social, a partir del análisis de habla consensual (definido previamente a través de la descripción del modelo ideal de comunicación) como base para el análisis de los modos deritativos (estratégicos) y defectivos (deformados) de habla.

Este tipo de investigación se apoya en las ciencias reconstructivas, que se sirven de la pragmática universal, que, en el caso de los actos de habla, permiten establecer las reglas que sitúan las oraciones en cualquier acto de habla. Son pues, ciencias empíricas, pero no empírico analíticas o nomológicas, es decir, se trata de la reconstrucción explícita, sistemática, de un conocimiento implícito, preteórico; por tanto las hipótesis reconstructivas, más que falsar, lo que permiten es hacer el saber preteórico explícito.

Estas reconstrucciones racionales plantean, necesariamente, una pretensión esencialista, dado que si son verdaderos los enunciados, tienen que corresponder precisamente a aquellas reglas que operan en el ámbito objetual, es decir, a las reglas que efectivamente determinan la producción de las estructuras superficiales.

A partir de aquí, el análisis que Habermas hace de los actos de habla, será limitado a los actos de habla institucionalmente no ligados, es decir, actos de habla que no presuponen instituciones particulares, sino que pueden encajar en toda una serie de marcos institucionales. Y así, deja fuera, los actos de habla institucionalmente ligados, es decir, jurar un cargo, desposar, nombrar, condenar... cuya aceptabilidad de sus actos de habla, está basada en reglas o normas, que constituyen un presupuestos del tipo de acto en cuestión.

\section{CONDICIONES MATERIALES PARA REA- LIZAR EL ANÁLISIS DE LOS MATERIALES DE LAS ENTREVISTAS Y DE LOS TEXTOS DISPONIBLES.}

Una cuestión clave que nos informa sobre la relevancia de la construcción de categorías, estriba en que los conceptos sociológicos son los denominados constructos de segundo nivel, elaborados a partir de los de primer nivel y éstos a su vez, son aquellos mediante los que los actores sociales han pre- estructurado ya la realidad social, con anterioridad a la investigación científica de ésta (McCARTHY, 1987.). Así, si entendemos que la acción social en que vienen mediados los esquemas interpretativos de los actores sociales, no puede ser captada independientemente de éstos, necesariamente habrá que partir de los constructos de primer nivel, para la configuración de los de segundo nivel. Es decir, las afirmaciones categoriales de naturaleza teóricoformal, que definen los marcos sociológicos, se formulan a partir de los elementos procedentes de la vida de la comunidad social.

Habermas señala las diferencias entre un lenguaje puro y un lenguaje natural. El lenguaje puro podría quedar definido en su sentido más amplio a través de reglas de constitución metalingüísticas. Sin embargo, el lenguaje natural, considera que desafía todo intento de "reconstrucción formalmente rigurosa, a causa de su "reflexividad"; incorpora en si formas no verbales de expresión (acciones y expresiones de vivencias) a través de las cuales es interpretado. En este sentido, el lenguaje ordinario es su propio metalenguaje" (McCARTHY, 1987, p.96). Por esa razón entiende que esa forma de vincularse lenguaje y práctica, es lo que nos permite el entendimiento mutuo en la práctica de la vida. Ello implica que el investigador hermenéutico sólo puede interpretar aquellos textos que tienen su origen en el contexto al cual pertenece.

En orden a ello, señala una restricción para los investigadores en solitario, dejando tan sólo una puerta abierta, en donde puede considerarse que "a lo sumo, de los intérpretes y traductores, de los científicos, (...), sí que puede decirse que cuando trabajan reflexivamente en ideas, valores y formas simbólicas de expresión, se están ocupando de objetos culturales" (HABERMAS, 1987 b, pág. 313). Por lo tanto, si de lo que se trata es identificar inicialmente las situaciones naturales de comunicación de las que no lo son, para identificar fines y saber preteórico, a partir de reconstrucciones racionales entonces, necesariamente, el investigador no tendrá más papel que el de ser un participante más dentro del contexto comunicativo.

Comentando esta idea de Habermas, el propio McCarthy señala que las condiciones que se producen en las situaciones de lo que él llama inefablemente individual, no pueden ser expresadas a través del análisis de la comunicación lingüística, ni tam- 
poco en la interacción simbólica, porque las biografías personales tienen un trasfondo concreto que determinan su sentido específico en expresiones y acciones particulares. $Y$ añade que,

"Las interpretaciones de las interpretaciones de la realidad se mueven en un nivel distinto que el de las interpretaciones de la realidad; en términos semánticos, los enunciados sobre enunciados son de orden distinto que los enunciados sobre hechos. Por otro lado, los contenidos semánticos tradicionales, que son los objetos de la investigación hermenéutica, son, a la vez, símbolos y hechos. Por lo tanto la interpretación es, también a la par, análisis empírico y análisis conceptual. Se dirige a los elementos de un mundo constituido mediante el lenguaje ordinario y a las propias reglas "gramaticales" que constituyen ese mundo" (McCARTHY, 1987, p.98).

y es precisamente el lenguaje ordinario, el terreno de esa intersubjetividad que hace posible la interacción y el entendimiento entre los individuos.

De manera que así expresadas las condiciones de la teoría crítica de la sociedad y el conocimiento desde el que es posible el acercamiento a cada tipo de interés, la coherencia entre la tarea iniciada y las condiciones materiales para la búsqueda de las razones, que no necesariamente tengan porque reflexionarse desde una perspectiva hermenéutica, pueden ser planteadas a partir de una búsqueda iluminativa. Como no se trata de una labor no reactiva, "las perspectivas de quienes se hallan ya incluidos en la situación" (CARR y KEMMIS, 1988, pág. 45), deberán ser representadas con la máxima fidelidad posible, de manera que el interés que quede en el sustrato del trabajo de indagación, se mueva en el espacio emancipatorio, incorporando tanto el técnico como el práctico, sin renunciar a la crítica social.

$Y$ recogemos una cita de Habermas que en el marco de la investigación, adquiere un sentido concreto que nos informa sobre lo que debiera ser esta tarea, cuando pretendemos abordarla desde la perspectiva crítica porque,

"La palabra "crítica" hace referencia aquí a una unión de competencia y capacidad de aprendizaje, que permita tanto una relación escrupulosa con un saber especializado considerado a modo de ensayo como una disposición, basada en una buena información y en la sensi- bilidad hacia el contexto, para la resistencia política contra conexiones funcionales del saber practicado que puedan ser sospechosas" (HABERMAS, 1987a, p. 358).

lo que aparentemente podría originar una duda sobre la metodología, dado que existe la posibilidad de tender hacia la definición de esta metodología como una enorme vaguedad. No es sólo un marco de intenciones lo que se apunta como red de identificación conceptual, sino que se persigue unas fundamentaciones con carácter universal, recogidas a partir de la comprensión de las orientaciones racionales de acción desde la reflexividad, abierta a los participantes efectivos del entorno donde se trabaja, en el que el investigador es participante virtual del mismo. Para el investigador su "única pretensión plausible de objetividad deriva del carácter reflexivo de su participación" (McCarthy, 1987, p., 453), y la expresión escrita del informe obtenido a partir del examen de reconstrucción racional "tiene por objeto revelar el sistema de reglas subyacentes a la producción de configuraciones simbólicas provistas de sentido. Su meta no es una paráfrasis o una traducción de un significado originalmente oscuro, sino un conocimiento explícito de reglas y estructuras cuyo dominio subyace a la capacidad que tiene un sujeto de generar expresiones con sentido" (McCarthy, 1987, p., 320). De modo tal, que nos encontramos así dentro del ámbito de pensamiento, que podremos encuadrar dentro de las ciencias empíricas, pero de modo diferenciado al del ámbito de las ciencias de las naturaleza, es decir, nos encontramos dentro del ámbito de las ciencias reconstructivas de carácter social.

El análisis de las expresiones de la comunicación con el procedimiento mayéutico como señala el propio Habermas, interroga una y otra vez al sujeto, a partir de relaciones de semejanza, contrastes, paráfrasis y ejemplos, de modo tal, que la relación de las teorías reconstructivas con el conocimiento cotidiano, no difieren de las ciencias empíricoanalíticas como señala McCarthy (1978). Veamos que si dentro de las ciencias empíricoanalíticas, lo que se hace es refutar el conocimiento preteórico de que se dispone a partir de los ámbitos objetuales, para ser sustituido por explicaciones teóricas correctas, aunque con carácter provisional hasta nueva demostración, en el terreno de las ciencias reconstrucctivas 
las hipótesis hacen explícito el saber preteórico y su relación con sus ámbitos objetuales.

\section{POSIBILIDADES PARA UNA TEORÍA RECONSTRUCTIVA DE LOS ACTOS DE HABLA}

Refiriéndonos en adelante a los actos de habla no institucionalizados, Habermas señala que en el acto de emisión, el hablante pone la oración en relación con la realidad, al tiempo que plantea pretensiones de validez de diferentes tipos: externa, interna y normativa. En definitiva, el hablante, en su acto de emisión pretende que lo que enuncia es verdadero, con intención veraz y correcto o adecuado; tres tipo de pretensiones de validez, que desde la perspectiva inmanente al lenguaje, la única pretensión es la inteligibilidad. Las otras pretensiones de validez, ponen la emisión del hablante, en relación con órdenes de realidad extralingüísticos.

Así, a partir de este esquema de los actos de emisión, para Habermas es factible el análisis de la competencia comunicativa, que exige una habilidad del hablante, no sólo para producir oraciones gramaticalmente correctas, sino para seleccionar el contenido proposicional, cuando se trata de reflejar una experiencia; expresar su intención sobre lo que piensa $\mathrm{o}$, finalmente, para realizar actos de habla que cumplan normas reconocidas o correspondan a autoimágenes aceptadas.

Esta es una concepción pragmática universal en la que basa sus líneas de investigación que reflejan al tiempo, las funciones pragmáticas del habla que se relacionan con la realidad. Estas funciones pragmáticas son: representativa, expresiva o interactiva. La fuerza ilocucionaria de los actos de habla consiste en la confianza del oyente, en la seriedad del compromiso indicado por el hablante, que no tiene por qué ser simplemente resultado del poder de sugestión, puede tener una base racional.

Estas pretensiones de validez llevan implícitas las obligaciones inmanentes a los actos de habla, que pueden cumplirse en dos niveles: inmediatamente o mediatamente.

Tanto el análisis experimental, como la revisión de los discursos teórico o práctico, así como las secuencias de acciones consistentes, partirán de la revisión/reflexión de los actos de habla, considerando la obligación que cada tipo de estos, asume la problematización que supone el incumplimiento de esa obligatoriedad. Estos tipos ya identificados, se pueden agrupar como sigue:

- Actos de habla constatativos. En su pretensión de verdad, el hablante asume una obligación inmamente al acto de habla, de aducir razones. La verdad problematizada, puede convertirse en objeto de un discurso teórico, si una vez que se ha recurrido a la fuente experimental en que basa el hablante su certeza, de que el enunciado es verdadero, no ha disipado las dudas ah hoc.

- Actos de habla regulativos. En su pretensión de rectitud o de adecuación, el hablante asume una obligación inmanente al acto de habla, de aducir justificaciones. Si la justificación inmediata no es capaz de disipar una duda planteada ad hoc, podemos pasar al discurso, y aquí se trataría del discurso práctico.

- Actos de habla representativos. En su pretensión de sinceridad o veracidad, marcamos la distinción entre el sí mismo real y las expresiones en que ese sí mismo aparecen.

En esencia, la teoría de la competencia comunicativa, se basa en la reconstrucción de la base normativa del habla como sistema de pretensiones de validez universales y necesarias, atendiendo al principio básico de que el lenguaje no puede ser comprendido con independencia del entendimiento, sin el que no podríamos acceder al lenguaje mismo. Los resultados del análisis que se siguen de esta noción de teoría comunicativa, en la que entran en consideración, tanto actos de habla, como acciones no-verbales, tiene para Habermas la finalidad de suministrar la base para una investigación social. El presupuesto básico que descansa en esta teoría comunicativa, es el consenso generado a partir del recíproco planteamiento de los hablantes, del mutuo reconocimiento de cuatro tipos de validez.

La acción comunicativa se vendrá abajo, en tanto se cuestionen alguna de sus pretensiones de validez, y su clarificación podrá realizarse de diferente modo, según sea el tipo de pretensión de validez cuestionada.

Si lo que se pone en cuestión es la inteligibilidad, sólo se restablecerá la comunicación si se clarifican los malentendidos, pudiendo superarse este tipo de perturbaciones, a partir de explicaciones, paráfrasis, traducciones o descripciones semánticas. 
Si lo que se pone en cuestión es la verdad de lo que uno dice, entonces este tipo de perturbación puede resolverse, suministrando nuevas informaciones, citando autoridades o experiencias relevantes.

Si lo que se pone en cuestión es que el contenido proposicional no es verdadero de un modo tan fundamental que quede interrumpida la comunicación, entonces las partes pueden tomar caminos dispares y recurrir a la fuerza o iniciar una rivalidad estratégica. Pero en todo caso podrán recurrir a la comunicación en un nivel diferente, esto es, el nivel del discurso teórico, donde una vez problematizada la verdad, se considerarán los contenidos proposicionales problematizados como verdades hipotéticas, quedando así, sometidas a la fuerza de argumentaciones y contraargumentaciones. El paso siguiente será de nuevo el establecimiento de la garantías de ser sinceros, haciendo evidente la buena fe, tratando de que el consenso deje de estar amenazado.

Si lo que pone es cuestión es alguno de las normas aceptadas, la perturbación originada puede resolverse dentro del contexto de interacción, recurriendo a normas establecidas, considerando que estas hubieran podido ser malinterpretadas, o a valores reconocidos.

La base consensual no queda menos perturbada si alguna de las partes interactuantes, es acusada por otra de las partes poniendo en duda la rectitud de sus intenciones. En este caso para que la comunicación pueda reestablecerse, es necesario hacerse mutuamente evidente su buena fe, a partir de la muestra de garantías, acciones consistentes, asunción de responsabilidades, disponibilidad a sacar consecuencias, etc.

Esta forma de clasificar los actos de habla de modo que se vinculen a unas pretensiones de validez, conduce a Habermas a plantear que la comunicación en el contexto interactivo, puede salir de un contexto de acción, y pasar a una situación discursiva, donde los participantes se someten a la "coacción no coactiva del mejor argumento" (McCARTHY, 1987, p. 338), quedando el discurso como "la condición de lo incondicionado" (Idem.). Así justifica el discurso argumentativo, que no dejando de ser una idealización, ha estado presente históricamente en nuestra tradición, en toda tentativa de institucionalizar "modos discursivos de exa- men de ciertos tipo de pretensiones de validez" (Ibídem).

Para Habermas el discurso teórico, además de ser condición de una adecuada teoría de la verdad, suministra claves para la estructura del discurso práctico, atendiendo que en ambos tipos de discursos -aunque radicalmente diferentes en la lógica de su argumentación-, la estructura y supuestos son las mismas. A las pretensiones de validez, se le objetan a Habermas véase Habermas (1988) págs. 398415).

En la base de las aclaraciones de Habermas, señalaremos que para nuestra guía de análisis, nos servirá su consideración de que el concepto de la acción comunicativa, ofrece la posibilidad de iluminar el saber implícito de que penetra en los procesos cooperativos de interpretación.

La descripción que plantea Habermas de los tipos puros de interacciones mediadas lingüísticamente es la siguiente:

- PERLOCUCIONES IMPERATIVAS. En lo que respecta a los elementos pragmáticos, Habermas considera que: cumplen la función del lenguaje de influencia sobre un oponente. Su acción esta orientada hacia el éxito. Su actitud básica es objetivante.

- CONSTATATIVOS. En lo que respecta a los elementos pragmáticos, cumplen la función del lenguaje de exposición de estados de cosas. Su acción esta orientada hacia el entendimiento. $\mathrm{Su}$ actitud básica es objetivante.

- REGULATIVOS. En lo que se refiere a los elementos pragmáticos, cumplen la función del lenguaje del establecimiento de relaciones interpersonales. Su acción esta orientada hacia el entendimiento.

- EXPRESIVOS. En lo que a los elementos pragmáticos se refiere, estos cumplen la función del lenguaje de presentación de uno mismo. Su acción esta orientada hacia el entendimiento.

Todos ellos, Habermas los identifica con los tipos puros de interacciones mediadas lingüísticamente (HABERMAS, 1988), considerando que pueden aproximarse gradualmente a las situaciones naturales de modo que sea posible servirse de ellos, para proceder al análisis reconstructivo, y donde la tarea consistirá en "ir desmontando de forma controlada las fuertes idealizaciones a las que se debe 
el concepto de acción comunicativa" (HABERMAS, 1988, p. 421).

Esta tarea parte de las siguientes consideraciones:

- paralelamente a los modos fundamentales, existen otras fuerzas ilocucionarias, propias de cada cultura y lenguas particulares que dan lugar a interacciones posibles plasmadas y estandarizadas.

- al tiempo que existen las formas estándar de los actos de habla, existen otras formas de realización lingüística de habla.

- además de los actos explícitos, se produce expresiones de carácter implícito, en las que la comprensión del oyente, está en relación al contexto particular donde se ha producido. Es el caso de emisiones abreviadas, que se pueden acompañar de efectos extraverbales.

- Además de los actos de habla directos, tienen lugar expresiones indirectas o ambiguas, en las que su comprensión se infiere de su contexto de origen.

- el análisis se hace extendible a las secuencias de actos de habla, a textos o diálogos, haciendo posible el establecimiento de las implicaciones conversacionales.

- en el análisis, hay que considerar de igual modo, tanto las actitudes objetivantes y de conformidad con las normas, derivadas de los actos de habla constatativos, regulativos y normativos, como la actitud realizativa, que de los actos de habla se deriven que hacen referencia a un mundo objetivo, social o subjetivo.

- este análisis, además de considerar el acto de habla propiamente, en cuanto a los procesos de entendimiento se refiere, considera también el efecto de la acción comunicativa, en el sentido de búsqueda de si se ha producido la coordinación consensual de los planes de acción de los participantes individuales, lo que nos pondría sobre la pista de otros efectos de carácter perlocucionario.

- así también, el análisis considera los mundo de la vida de los participantes, en tanto que trata de conocer el saber de fondo de los participantes, dado que ello forma parte de las interpretaciones que todos realizan.

Este distanciamiento de Habermas, de la situación de actos de habla ideales, permite realizar la distinción de cuándo se producen:

a) situaciones naturales de entendimiento. b) situaciones orientadas al éxito.

y esta distinción, la señala en orden a la clarificación de que igual que en situaciones estratégicas tienen lugar ilocuciones, en los contextos de acción comunicativa, también pueden aparecer perlocuciones, ya que los procesos cooperativos, pasan por varias fases.

\section{UNA POSIBILIDAD DE APLICACIÓN}

Los argumentos expuestos permiten plantear la modalidad metodológica de estudios de casos a partir de una exploración de campo, tomando notas observacionales, planteando entrevistas abiertas y distribuyendo un amplio cuestionario además de contrastar opiniones con miembros integrantes de ese entorno institucional enfermero donde se investiga. La aplicación de la teoría reconstructiva habermasiana permite, contando con el conjunto de informaciones obtenidas, las normas y reglas internas institucionales, las formalizaciones teóricas, etc., establecer dentro de los diálogos una serie de actos de habla problematizados, es decir, aquellos que no obedezcan a la pretensión de validez que el hablante supone. Lógicamente las personas en una charla abierta, deben ser informadas del tipo de análisis que sus afirmaciones permitirán. Conjuntamente con la disposición de reglas, normas y teorías que nos permitirán contrastar los diferentes tipos de emisiones y estudiar la naturaleza proposicional de estos.

La fuerza de las argumentaciones y contraargumentaciones, en un análisis posterior, nos dará paso a una serie de conclusiones previas, en algunos casos, que permitan nuestra propia construcción teórica en la definición del problema que estudiemos.

Lo cierto es que, como método cualitativo, el hecho de ofrecernos la posibilidad de establecer distingos entre el discurso práctico y discurso teórico, salvando el problema que en los estudios fenomenológicos plantean las percepciones que para Habermas serían los actos de habla expresivos, nos encontramos con una clasificación de los actos de habla, que con toda su carga empírica y racional, nos permite distinguir las emisiones perlocucionarias de las ilocucionarias y nos acredita las pretensiones de validez a las construcciones teóricas que de ello se derivarían, al tiempo que abren una nueva posibilidad de mejora del entendimiento de los sujetos de través de la coordinación consensual de 
los planes de acción de los sujetos. El informe final, que tendrá en buena lógica formato de estudio emergente, será de naturaleza ideográfica como corresponde a los estudios de casos, dentro de la gama que acoge el paradigma naturalista y en él se expresará el recorrido teórico que motivó el estudio del caso, los formatos de recogida de datos e información, el análisis de los textos de acuerdo con los formatos cualitativos y las construcciones teóricas que de ello se pueda desvelar.

\section{BIBLIOGRAFÍA}

Ardoino, J. (1980) Perspectiva política de la educación. Trad. por A. Casais. Narcea, Barcelona

Carr, W; Kemmis, S. (1988): Teoría crítica de la enseñanza. Trad. por J.A. Bravo. ed.orig. 1986. Martínez Roca, Barcelona.

Habermas, J. (1987) Teoría y praxis.Estudios de filosofía social. Trad. por Mas Torres, J. y Moya Espi, C. ed.original 1963. Tecnos, Madrid.
Habermas, J. (1987b) Teoría de la acción comunicativa II. Crítica de la razón funcionalista. Trad. por M. Jiménez. ed. original 1981. Taurus, Madrid.

Habermas, J; Bermas, J. (1988) Teoría de la acción comunicativa I. Racionalidad de la acción y racionalización social. Trad. por M.Jiménez. ed.original 1981. Taurus, Madrid.

Habermas, J. (1990a) La lógica de las ciencias sociales. Trad. por M.Jimenez. $2^{\mathrm{a}}$ ed. castellano. ed. original 1982. Tecnos, Madrid.

Habermas, J. (1986a) Conocimiento e interés. Trad. por M.Jimenez, J.F.Ivars y L.M.Santos. ed.original 1968. Taurus, Madrid.

Habermas, J. (1986b) Ciencia y Técnica como ideología. Trad. por M.Jimenez y M. Garrido. ed.original 1968. $1^{\text {a }}$ reimpresión. Tecnos, Madrid.

McCarthy, T. (1987) La Teoría Crítica de Jürgen Habermas. Tecnos, Madrid.

Esquema habermasiano de los tipos puros de interacciones mediadas lingüísticamente

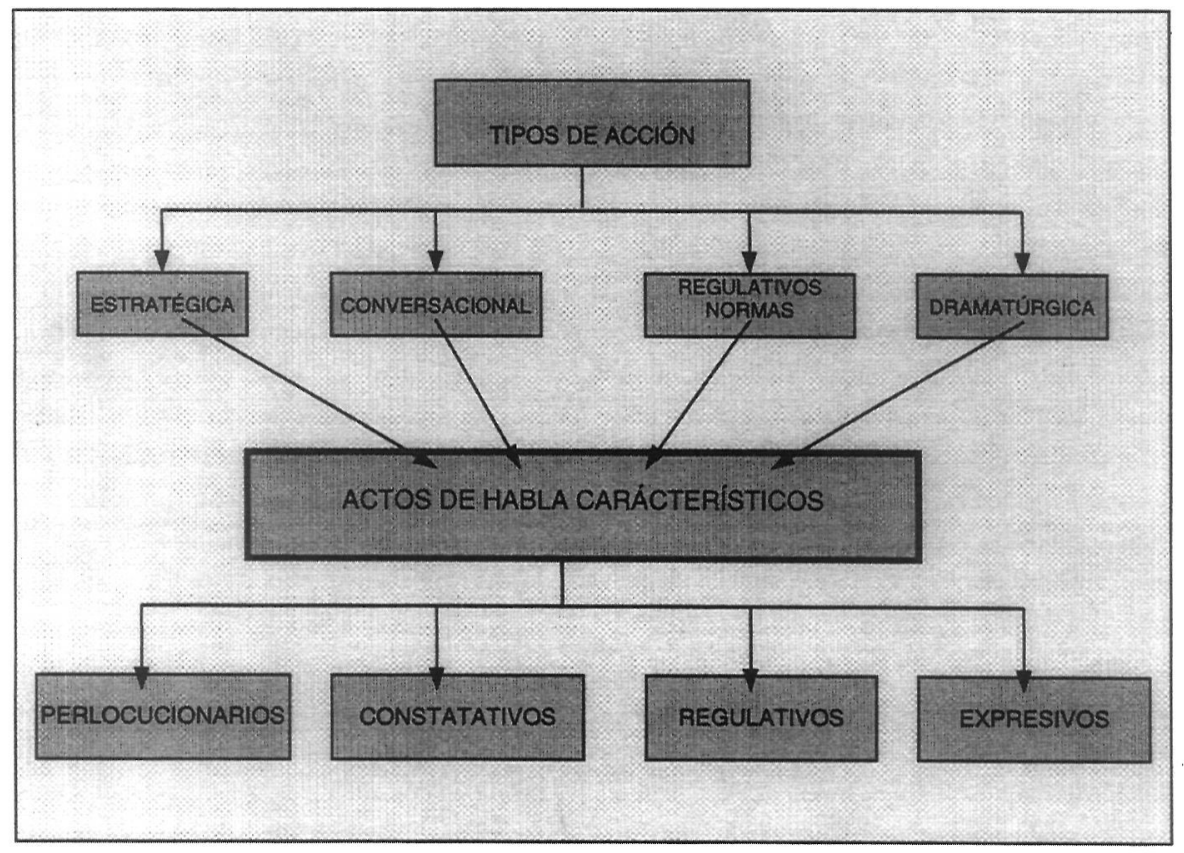

